\title{
Anabases
}

ANABASES Traditions et réceptions de l'Antiquité

3 | 2006

Varia

\section{Le projet D@GR (Dictionnaire des antiquités grecques et romaines en ligne)}

\section{Geneviève Rives-Gal}

\section{(2) OpenEdition}

1 Journals

Édition électronique

URL : http://journals.openedition.org/anabases/3614

DOI : 10.4000/anabases.3614

ISSN : 2256-9421

Éditeur

E.R.A.S.M.E.

\section{Édition imprimée}

Date de publication : 1 mars 2006

Pagination : 252-258

ISSN : 1774-4296

\section{Référence électronique}

Geneviève Rives-Gal, « Le projet D@GR (Dictionnaire des antiquités grecques et romaines en ligne)», Anabases [En ligne], 3 | 2006, mis en ligne le 01 janvier 2012, consulté le 20 octobre 2019. URL : http:// journals.openedition.org/anabases/3614 ; DOI : 10.4000/anabases.3614

Ce document a été généré automatiquement le 20 octobre 2019

(c) Anabases 


\title{
Le projet D@GR (Dictionnaire des antiquités grecques et romaines en ligne)
}

\author{
Geneviève Rives-Gal
}

1 C'est Aline Rousselle, professeur d'histoire romaine à l'Université de Toulouse-Le Mirail, qui la première dès 2001, enthousiasmée par les innombrables possibilités offertes par le nouvel outil de communication Internet, a exprimé l'idée de mettre en ligne et de rajeunir le Dictionnaire des antiquités grecques et romaines. Cette séduisante proposition fut considérée alors comme un rêve presque irréalisable compte tenu des difficultés techniques à vaincre ${ }^{1}$ et de l'absence de financement pour y parvenir. Pour des raisons de santé, Aline Rousselle dut malheureusement cesser son activité parmi nous en 2002, et cette affaire en serait certainement restée au stade du songe faute d'un véritable dossier définissant des objectifs et proposant un cadre pour leur réalisation.

2 Ce n'est qu'au printemps 2004 que nous avons mis rigoureusement en forme le projet D@GR en étroite collaboration avec la Mission «Innovation Technologique et Multimédia » de l'UTM $^{2}$ (avec Françoise Adreit ${ }^{3}$ et Bernard Pellefigue ${ }^{4}$ ), assistée par le Centre Audiovisuel et Multimédia de l' UTM (en la personne de Nathalie Michaud ${ }^{5}$ ), et soutenue par l'ensemble des équipes de recherche sur l'Antiquité de notre université (CRATA ${ }^{6}$, ERASME ${ }^{7}$ et $\mathrm{UTAH}^{8}{ }^{8}$. Il s'agissait de répondre à un appel d'offre du ministère de la Recherche concernant la «Création de produits de médiation scientifique en libre accès sur l'Internet ».

3 C'est la préparation de ce dossier qui nous a permis de nouer les premiers contacts extérieurs à notre université et a donné une impulsion décisive au projet. Nous allons en synthétiser ici le contenu en présentant tout d'abord le Dictionnaire des antiquités afin d'expliquer tout l'intérêt de sa mise en ligne ainsi que la nécessité de son enrichissement et de sa réactualisation. 


\section{Pourquoi le Dictionnaire des antiquités grecques et romaines?}

4 Bien que tous les antiquisants connaissent cet ouvrage, il nous parait opportun d'en rappeler ici les principales caractéristiques qui en expliquent la valeur et justifient le lancement du projet.

\section{L'ouvrage et ses concepteurs}

$5 \quad$ Le titre complet de l'ouvrage est Dictionnaire des antiquités grecques et romaines, d'après les textes et les monuments, contenant l'explication des termes qui se rapportent aux mours, aux institutions, à la religion, et en général à la vie publique et privée des anciens.

6 Il regroupe environ 2600 notices assorties de notes infrapaginales et d'illustrations (8664 pages réparties en cinq tomes et dix volumes - dont un volume de tables ; près de 6000 illustrations). L'ouvrage a été conçu, pour répondre à une demande de Louis Hachette (1800-1864), par un médecin ami d'Émile Littré (1801-1881), féru d'édition et de médecine antique, Charles-Victor Daremberg (1817-1872). C'est ce savant qui en élabora le plan et en choisit les premiers auteurs-contributeurs, bien qu'il ne soit intéressé que très marginalement par l'histoire ancienne, en dehors des textes relatifs à la médecine. Il mourut en 1872 avant la publication, en $1873^{\circ}$, chez Hachette du premier fascicule, mais des matériaux considérables avaient déjà été recueillis si bien que l'œuvre put être poursuivie, sans grandes modifications de plan, par Edmond Saglio ${ }^{10}(1828-1911)$ jusqu'à sa mort. La coordination de ce travail fut reprise par Edmond Portier ${ }^{11}$ (1855-1934) aidé par son gendre Georges Lafaye; la publication complète fut achevée en 1919. L'ouvrage a été réédité deux fois au $\mathrm{xx}^{\mathrm{e}} \mathrm{s}$. : à Paris par Hachette en 1962-1963 et par l'Akademische Druck- und Verlagsanstalt de Graz (Autriche) en 1969.

7 Mais ces renseignements chronologiques et l'énormité de l'œuvre ne suffisent pas pour expliquer tout l'intérêt de l'ouvrage qui émerge surtout des contextes culturel et politique de la fin du XIX ${ }^{e}$ s. et du début du $\mathrm{Xx}^{\mathrm{e}} \mathrm{s}$.

\section{Contexte de rédaction}

\section{L'émergence des Sciences de l'Antiquité}

Rédigé à partir du dernier quart du XIX ${ }^{\mathrm{e}}$ s., le Dictionnaire des Antiquités est le reflet d'une époque qui voit émerger le concept de sciences de l'Antiquité. Jusqu'à la fin du XVIII ${ }^{e}$ s., en effet, la curiosité des savants s'était trop souvent satisfaite des propos des auteurs antiques; les antiquaires, bien que conscients de l'intérêt historique du matériel en provenance de l'Antiquité (œuvres d'art, monnaies, objets divers, textes épigraphiques) ${ }^{12}$, et malgré le développement d'une légitime méfiance vis à vis des sources littéraires, se contentaient au bout du compte d'accepter les données de ces dernières ${ }^{13}$. Ce n'est qu'au cours du xix $s$. que l'approche de l'Antiquité se renouvela totalement grâce à l'apparition lente d'une archéologie scientifique ${ }^{14}$ et à l'affirmation de disciplines dérivées telles que l'épigraphie ${ }^{15}$, la numismatique ${ }^{16}$ et la céramologie ${ }^{17}$. Dès lors on commença systématiquement à confronter les informations fournies par les textes à celles procurées par le matériel archéologique tout en forgeant l'apparat 
critique des uns et des autres et en développant des méthodes d'investigation convenables pour chaque type de matériel en fonction des questions auxquelles on entendait répondre. C'est sur cet ensemble de démarches que se fondent les Sciences de l'Antiquité telles que nous les connaissons aujourd'hui et c'est dans cette perspective qu'il faut replacer le Dictionnaire des Antiquités, mais, au-delà des exigences scientifiques nouvelles, les concepteurs et les auteurs de cet ouvrage entendaient, en même temps, satisfaire à des impératifs d'un autre ordre.

La concurrence entre les nations d'Europe occidentale: les sciences de l'Antiquité, référence et partie du prestige national

L'Antiquité jouissait à cette époque d'un immense prestige et sa connaissance était l'apanage et la référence culturelle commune des élites sociales d'Europe occidentale. Assimilé à une vertu, le savoir relatif à l'Antiquité, permettait à ceux qui le détenait de légitimer la supériorité de leur position sociale; de même, la présence dans un pays d'une communauté d'érudits antiquisants de plus ou moins grande qualité était le reflet de son niveau de civilisation et devait donc décider de son aptitude à peser dans le concert international voire à dominer le monde. C'est pour cela que les sciences de l'Antiquité furent mobilisées dans la compétition entre les nations d'Europe occidentale et devinrent l'un des champs dans lesquels s'exprimèrent les patriotismes rivaux, en particulier la rancœur franco-allemande développée en Allemagne depuis l'époque napoléonienne et exacerbée en France par la défaite de 1870 et le dur traité de 1871.

C'est dans ce double contexte, celui de l'essor des Sciences de l'Antiquité et celui des rivalités nationales, principalement celle de la France et de l'Allemagne, qu'il faut replacer la rédaction du Dictionnaire des Antiquités. En élaborant les structures de cet ouvrage, dès les années 1860, les Français entendaient faire pièce à l'avance acquise par les Allemands dans de nombreux domaines relatifs à l'Antiquité (philologie, épigraphie, reconnaissances de vases et d'œuvres d'art, etc.) ; le sentiment de frustration consécutif à la défaite ne fit sans doute qu'accroître le désir de déstabiliser l'adversaire allemand dans un de ses champs d'excellence afin d'entamer la Revanche. N'avait-on pas prétendu que la défaite était la conséquence du délabrement intellectuel de nos élites et qu'en conséquence il fallait faire du relèvement des études scientifiques une préoccupation nationale; les sciences de l'Antiquité devaient en conséquence intervenir dans ce redressement salvateur.

\section{Contenu scientifique : supplanter l'adversaire en fournissant quantité et qualité}

11 Pour convaincre de la précellence des sciences de l'Antiquité françaises, il fallait fournir le plus et le meilleur; de ces aspirations découlent les options prises par les concepteurs de l'ouvrage ${ }^{18}$.

12 Tout d'abord, on peut penser que le choix pour la forme dictionnaire s'explique par la vogue de ce genre d'ouvrages ${ }^{19}$, mais répond surtout au désir d'être exhaustif (c'est l'effet masse) tout en offrant une commodité d'utilisation. Plus qu'un dictionnaire, d'ailleurs, notre ouvrage est une encyclopédie de l'Antiquité qui, de manière originale, exclut de ses titres de notices les événements historiques, les lieux et les personnages. Cette dernière démarche provient probablement de la volonté, d'une part d'exploiter les ressources fournies par l'archéologie et, d'autre part, d'offrir aux archéologues un outil performant en leur permettant d'accéder rapidement aux données textuelles. On 
notera aussi que les Français avaient devancé leurs adversaires en implantant un institut à Athènes (1846) et qu'ils entendaient profiter de cet avantage qui fut renforcé après les initiatives d'Albert Dumont lorsqu'il prit la direction, en 1875, de l'École française d'Athènes. C'est pour cela que les auteurs sollicités par Daremberg et ses successeurs étaient très fréquemment issus de cet établissement.

Ils fournirent dès l'origine des articles extrêmement complets faisant le point des connaissances d'alors sur le sujet en confrontant les différentes sources d'information conformément aux nouvelles pratiques historiques. Les notices furent largement agrémentées de plans de sites et de monuments, de reproductions de monnaies et d'inscriptions. L'abondance d'une iconographie d'excellente qualité, ce qui était largement une nouveauté, contribua sans doute au succès.

Le lancement de ce travail de longue haleine eut un immense retentissement dans les pays de langue française et largement au-delà. La réaction ne se fit pas trop attendre puisque, en 1886, les savants allemands ripostèrent en lançant la fameuse Realencyclopädie ; d'autres suivirent, en italien et en anglais.

\section{Le Dictionnaire aujourd'hui}

Un siècle plus tard, il est clair que de nombreux articles sont dépassés, néanmoins le Dictionnaire des Antiquités reste un ouvrage très consulté car il offre des renseignements irremplaçables sur l'univers matériel de l'Antiquité et rassemble dans les notes infrapaginales les références des sources littéraires relatives à chaque sujet. Toutefois son utilisation n'est pas ce qu'elle pourrait être car l'ouvrage, sans être très rare, est loin de figurer dans toutes les bibliothèques; d'autre part sa consultation reste fastidieuse, les volumes sont encombrants et lourds et l'on doit souvent passer de l'un à l'autre ce qui rend la recherche pénible.

16 C'est de tous ces constats (valeur patrimoniale, qualité et utilité, rareté et incommodité) que le projet Dictionnaire des Antiquités en ligne est né; il s'agissait de conserver et de valoriser cette œuvre monumentale en la rendant accessible au plus grand nombre tout en prenant en compte l'actualité scientifique.

\section{Le projet D@GR}

17 Tel qu'il a été conçu en 2004 pour répondre à l'appel d'offre ministériel, le projet prévoyait deux phases : une phase de numérisation et de mise en ligne avec élaboration de l'outil de consultation et une phase d'enrichissement et de révision progressive des articles sous forme de réécriture ou de commentaires à propos de points précis. Notre projet n'a pas été retenu par le ministère mais sa bonne position dans le classement a incité la Mission innovation technologique et multimédia à prendre à sa charge le financement de la première phase. Cette dernière est achevée depuis le mois de septembre 2005.

\section{Mise en œuvre et bilan de la première phase}

18 Pour réaliser la première phase, une série de volumes a été acquise afin de rendre possible le massicotage de l'ouvrage sans mettre en péril les exemplaires de notre bibliothèque universitaire. Après cette première opération, la numérisation a été 
effectuée automatiquement en mode image et en mode texte (utilisation d'un logiciel OCR permettant la reconnaissance de caractères) ${ }^{20}$. Le produit texte brut a été balisé physiquement (position des mots sur la page, style d'écriture) ce qui a permis de repérer les entrées pour obtenir un fichier xML seulement balisé sur celles-ci. Les neuf volumes d'articles sont désormais en accès gratuit sur Internet ${ }^{21}$. Nous offrons sur le site du D@GR ${ }^{22}$ deux possibilités de consultation: une recherche alphabétique traditionnelle et une recherche par mot dans le plein texte (avec possibilité d'avoir recours aux opérateurs $+,-, ?, *)$. Une loupe permet de lire plus commodément les notes (elle permet d'accéder à l'article original en haute définition - $300 \mathrm{dpi}$ ); on peut aussi voir les images sous forme de diaporama et accéder à l'article en mode texte (le texte ayant été reconnu par un procédé optique comporte des erreurs - $15 \%$; une note permet de prévenir les utilisateurs et leur recommande, en cas de problème, de se référer au texte original). Il est enfin possible de déposer librement des commentaires sur chaque article sous la surveillance d'un modérateur susceptible de supprimer les propos qui sortiraient du domaine de l'information scientifique. En marge de cette première phase, les techniciens pensent pouvoir offrir une possibilité de consultation sur les noms des multiples auteurs-contributeurs du dictionnaire.

\section{La deuxième phase (en cours de réalisation)}

L'objectif est de mettre en ligne progressivement les enrichissements (sources littéraires traduites, documents iconographiques et épigraphiques, bibliographies actuelles) et les révisions et/ou commentaires validés par le site (différents des commentaires libres et reconnaissables à l'affichage par la couleur de leur fond). La mise en œuvre de cette phase comporte deux aspects :

- un aspect technique qui comprend, d'une part, l'élaboration de l'outil servant de support aux enrichissements et aux révisions (création de fichiers "Révisions", "Bibliographie ", "Documents iconographiques ", "Sources littéraires ", "Documents divers » relatifs à chaque article et moyen de les atteindre à partir de l'article ouvert initialement) et, d'autre part, la mise au point du dispositif permettant le dépôt direct de tous ces éléments par quelques personnes habilitées ${ }^{23}$ qui organiseront leur collecte ou prélèveront parmi les commentaires libres ceux qui leur paraîtrons les plus pertinents, cela pour éviter d'avoir recours systématiquement à des techniciens et acquérir la souplesse nécessaire à la réalisation d'un tel projet. Ce support est en voie d'être achevé puisque une maquette de fonctionnement va être proposée au cours du premier trimestre 2006 ;

- un aspect scientifique: nous sommes en train de composer un comité de pilotage comprenant des chercheurs spécialisés dans les divers domaines couverts par le dictionnaire. Eux seuls pourront produire des enrichissements et commentaires validés par le site, solliciter d'autres auteurs-contributeurs (aussi bien des spécialistes confirmés que des jeunes chercheurs) et faire leur choix dans les commentaires ouverts à tous. Pour cela nous faisons appel à tous ceux qui sont intéressés par l'avancement de ce projet, chacun pouvant se charger d'un domaine de son choix (on peut nous contacter sur le site D@GR). Chaque membre du comité de pilotage pourra avoir accès au site pour déposer le fruit de sa collecte. 


\section{Bilan et perspectives}

La réussite de la première phase nous incite à l'optimisme. Non seulement les retours favorables ont été nombreux mais, en outre, nous avons compris que les possibilités offertes par la numérisation et l'accès au en plein texte donnait une dimension nouvelle à l'ouvrage puisque la recherche par mot permet d'accéder à des renseignements concernant les événements, les lieux et les personnages exclus de la liste des notices. Certes, nous avions subodoré l'intérêt de la numérisation et de la mise en ligne de cet ouvrage, mais l'usage dépasse nos espérances : les données peuvent être brassées très rapidement si bien que l'on peut mieux prendre en compte toutes les ressources contenues dans le dictionnaire et bien sûr, à l'inverse, on en perçoit mieux les insuffisances actuelles.

C'est pour remédier à cela que nous espérons pouvoir rapidement mettre en œuvre la deuxième phase; déjà une trentaine de contributeurs potentiels se sont déclarés et nous espérons que de nombreux autres vont s'empresser de nous rejoindre.

\section{NOTES}

1. En particulier la numérisation des volumes qu'il était impossible alors d'imaginer sans une manipulation compliquée et fatalement coûteuse.

2. http://www.univ-tlse2.fr/multimedia/

3. Directrice de la mission IT2M, maître de conférences en informatique, département de mathématiques-informatique, UFR Sciences Espaces Sociétés.

4. Éditeur de projets, ingénieur d'étude; il aide les auteurs de projets à concevoir et à réaliser leurs projets.

5. Chargée de production au CAM.

6. http://www.univ-tlse2.fr/rech/equipes/crata.html

7. http://www.univ-tlse2.fr/rech/equipes/erasme.html

8. http://www.univ-tlse2.fr/rech/equipes/utah.html

9. Avant 1877 , les premiers articles ont été publiés sous forme de fascicules.

10. Directeur du département des objets d'art du Musée du Louvre.

11. Conservateur en chef du département des antiquités gréco-romaines et orientales du Musée du Louvre, Edmond Pottier est à l'origine du Corpus vasorum antiquorum.

12. Par exemple, Charles-César BAUDELOT DE DAIRVAL fait paraître à Paris, en 1686, un ouvrage intitulé De l'utilité des voyages et de l'avantage que la recherche des antiquités procure au savant et JeanFoy VAILLANT publie pareillement à Paris, à partir de 1728, Arsacidarum imperium, sive regum Parthorum historia. Ad fidem numismatum accommodata, une histoire de l'Empire parthe fondée sur l'étude des monnaies.

13. Ceci s'explique en partie parce que les faux antiques (des monnaies en particulier) circulaient largement ce qui, bien entendu, leur ôtait toute valeur de preuve; de plus la tradition littéraire jouissait d'un immense prestige si bien que, lorsqu'il y avait désaccord entre les textes et les monnaies, on estimait qu'il était préférable de croire les premiers, comme l'explique H. NicolETPIERRE (Numismatique grecque, Paris, 2002, p. 47-48). 
14. Dans le domaine de l'archéologie l'activité devint très intense dans la deuxième moitié du XIX s. L'École française d'Athènes est fondée en 1846 pour des raisons plus politiques qu'archéologiques; celle de Rome n'est créée qu'entre 1873 et 1875 d'abord comme une dépendance de celle d'Athènes. En 1873, l'Institut archéologique de Berlin ouvrit une antenne à Athènes (Institut allemand d'Athènes) faisant perdre à la France son monopole. Pour réaffirmer sa présence active, l'École française se mit, à partir de 1877, à publier les informations recueillies lors de ses campagnes de fouilles devenues systématiques dans le Bulletin de correspondance hellénique.

15. Les premiers corpus épigraphiques furent constitués par des Allemands en particulier par August BОЕСКн à partir du deuxième quart $\mathrm{du} \mathrm{XIX}^{\mathrm{e}} \mathrm{s}$. Les érudits allemands avaient acquis une certaine avance en Europe dans ce domaine, ce qui rendait jalouses les autres communautés savantes.

16. Les premières grandes collections numismatiques royales ou princières se constituent dans la deuxième moitié du XVII $\mathrm{s}$, mais ce n'est qu'en 1836 que naît en France la Revue numismatique et seulement en 1865 la Société française de numismatique et d'archéologie.

17. C'est Eduard GERHARD (1795-1867) qui donne la première impulsion à l'étude scientifique des vases grecs. Son premier inventaire de vases grecs, les Auserlesene griechische Vasenbilder, hauptsächlich etruskischen Fundorts, est publié 1839.

18. Une étude historiographique plus approfondie mériterait d'être faite à ce sujet. P.-P. CORSETTI nous a spontanément suggéré une bibliographie sommaire : S. REINACH, «Edmond Saglio», Revue archéologique 1911 (2), p. 456-458 ; « Notice de M. Edmond Pottier [sur E. Saglio]», in DAGR 5 (1919), p. 3-8 (notice placée en tête du fascicule 46, paru en 1912) ; U. CHEVALIER, « Notice sur la vie et les travaux de M. Edmond Saglio ", Comptes rendus de l'Académie des Inscriptions et Belles-lettres 1913, p. 161-197 ; G. LAFAYE, «L'achèvement d'une œuvre française », Revue archéologique 1917 (1) 271-281.

19. Ils sortent au rythme de plusieurs par an à partir des années 1860 ; ils couvrent toutes les thématiques, des plus classiques aux plus spécialisées ; on peut citer : M.P. PoITEVIN 1860, Nouveau dictionnaire universel de la langue française; NADAL, 1861, Dictionnaire d'éloquence sacrée ; CHESNEL, 1862, Dictionnaire des armées de terre et de mer. Encyclopédie militaire et maritime; E. LITTRÉ, 1863, Dictionnaire de la langue française; J. CHENU, 1863, Encyclopédie d'histoire naturelle; Ch. DEZOBRY et Th. BACHELET 1863, Dictionnaire Général de Biographies et d'Histoire, de Mythologie, de Géographie ancienne et moderne; etc.

20. SICD (Cellule de numérisation 11, rue des puits-creusés, 31000 Toulouse) et Service imprimerie-numérisation (Université Toulouse II-Le Mirail).

21. Ces deux dernières étapes ont été réalisées par Hélène JOUGUET (chef de projet - DESs Archives et Images), Dounia CHEDDADI (révisions XML - stagiaire en licence mathématiques appliqués et sciences sociales) et Florent LARTET (développement informatique - étudiant en alternance, maîtrise IUP Nouvelles technologies de l'informatique pour l'entreprise).

22. http ://dagr.univ-tlse2.fr/sdx/dagr/

23. Ces personnes disposeront d'un identifiant et d'un code pour leur permettre d'accéder au site. 


\section{AUTEUR}

GENEVIÈVE RIVES-GAL

c_gal@club-internet.fr 